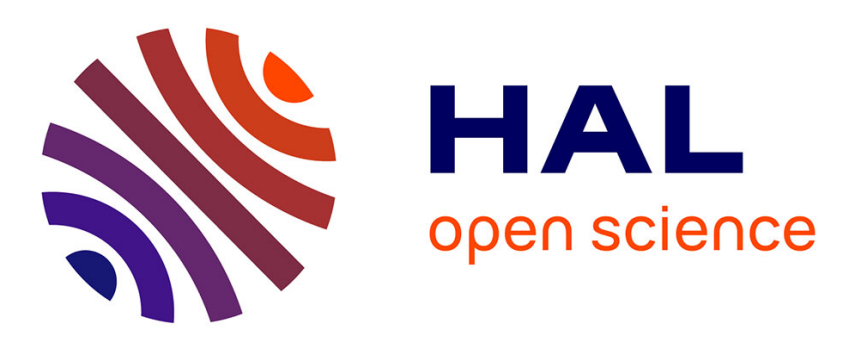

\title{
"Le vrai sanaani ". Note sur la perception des changements linguistiques à Sanaa, Yémen
}

\author{
Julien Dufour, Hanan Maloom
}

\section{To cite this version:}

Julien Dufour, Hanan Maloom. " Le vrai sanaani ". Note sur la perception des changements linguistiques à Sanaa, Yémen. Langage et Société, 2011, Villes du monde arabe: variation des pratiques et des représentations, 138, pp.97-105. 10.3917/ls.138.0097 . hal-01329860

\section{HAL Id: hal-01329860 \\ https://hal-amu.archives-ouvertes.fr/hal-01329860}

Submitted on 13 Oct 2016

HAL is a multi-disciplinary open access archive for the deposit and dissemination of scientific research documents, whether they are published or not. The documents may come from teaching and research institutions in France or abroad, or from public or private research centers.
L'archive ouverte pluridisciplinaire HAL, est destinée au dépôt et à la diffusion de documents scientifiques de niveau recherche, publiés ou non, émanant des établissements d'enseignement et de recherche français ou étrangers, des laboratoires publics ou privés. 


\section{"Le vrai sanaani ". Note sur la perception des changements linguistiques à Sanaa, Yémen}

\section{Julien Dufour}

Université de Strasbourg, julienetiennedufour@hotmail.com

Hanan Maloom

IREMAM et Université de Provence, hanan_maloom@yahoo.fr

\section{Introduction}

Les habitants de Sanaa, de toutes origines, expriment généralement une conscience vive de l'existence d'un parler sanaani. Partant de cette constatation, nous avons voulu savoir ce qu'ils entendaient par là, en leur posant directement la question. Comme on pouvait s'y attendre, les choses ne sont pas simples et, dans une certaine mesure, le sanaani, tel un arc-en-ciel, s'évanouit quand on s'en approche. L'enquête nous en apprend plus sur les positionnements identitaires que sur des caractéristiques linguistiques objectives.

Les remarques qui seront exposées ici s’appuient au premier chef sur une série d'entretiens réalisés à Sanaa par Hanan Maloom au cours de l'année 2010, auprès de quatorze habitants de Sanaa des deux sexes, âgés de 20 à 68 ans, nés à Sanaa et dont une partie au moins de la famille est originaire de cette ville; une famille toutefois est entièrement originaire d'Aden. Cette enquête, dont les circonstances actuelles ne facilitent pas l'approfondissement, ne permet pas encore de tirer des conclusions assurées. Elle permet tout de même de relever certains discours récurrents et d'émettre des hypothèses, qui resteront à confirmer. Il ne nous a pas semblé inutile d'exposer ces résultats imparfaits, dont l'intérêt réside surtout dans les questions qu'ils suscitent. Nous proposerons donc à partir du peu que nous savons un exposé qui s’appuie assurément sur des faits, 
des recherches et des constatations, mais dont on ne saurait honnêtement prétendre qu'il s'impose de façon automatique au terme d'une enquête impartialement menée auprès d'un échantillon représentatif.

Cet exposé laisse injustement de côté, faute de données, les populations issues du Sud au sens large, d'Aden à Taez. Cette dernière ville est depuis longtemps un relais pour les influences venues - en viennent-elles encore? - d'un Sud qui échappe largement à l'alternative

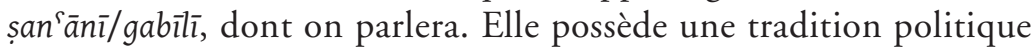
qui lui est propre et elle a montré - et payé fort cher - un engagement massif dans la contestation qui secoue le pays, ce qui peut lui conférer un crédit nouveau à l'échelon national. Or une partie importante des migrants installés à Sanaa vient de Taez et de sa région, en particulier de la Hujariyyah. C'est un des dialectes que l'on entend le plus dans les rues. On peut s'attendre à rencontrer dans cette immigration-là des problématiques ou des réactions un peu différentes.

\section{Le vieux parler de Sanaa}

Quelques précisions préalables. Sanaa, au début des années 1970, est une bourgade de 80000 habitants au maximum. Elle en compte aujourd'hui près de trois millions, principalement à cause d'une immigration provenant du Yémen entier, mais où l'arrière-pays de Sanaa est proportionnellement bien représenté (Stadnicki 2009 : 225). Stadnicki (2009: 142) estime qu'environ $70 \%$ des habitants de la ville sont nés ailleurs; le taux de croissance annuel avoisine les $10 \%$. Watson (2004) retrace les principales étapes de ce développement urbain.

Le dialecte traditionnel de Sanaa, celui de la vieille ville d'avant l'essor démographique, décrit par Rossi (1939), mais aussi celui, plus récent, que décrit Watson (1993), tout à fait dans la continuité du précédent, n'est pas d'un type fondamentalement différent de ceux pratiqués dans les campagnes avoisinantes. Il s'inscrit dans un ensemble dialectal, celui des hauts plateaux, dont les limites peuvent être délicates à fixer ${ }^{1}$, mais qui s'oppose clairement à d'autres ensembles: Tihāmah, région d'Ibb, de Taez, parlers du Yémen méridional et oriental. On n'est donc pas dans le cas où un parler citadin ancien représenterait une enclave dans une zone qui lui serait linguistiquement hétérogène. Sanaa, qui exerce sur sa région une forte attraction migratoire, se retrouve donc en position de capitale régionale linguistique, en plus d'être la capitale économique du Yémen.

1. À propos du classement des aires dialectales yéménites, voir Behnstedt (1985: 226), qui confirme les intuitions informées de Rossi (1938 : 244 sq.), et Watson (1994). 
Au vu des entretiens effectués, il apparaît que le sanaani vaut avant tout comme référence mythique. La majorité des informateurs disent spontanément qu'aujourd'hui plus personne ne parle le vrai sanaani, avant, parfois, d'ajouter que peut-être on peut encore trouver des gens qui le parlent. Ce qui implique, d'une part, qu'il existe un vrai sanaani (șan'āñ hagìigì, șan'ānī șudg, șan'āni-șan`ānī) et, d'autre part, que ce vrai sanaani est perdu. Le caractère sanaani ou non d'un parler est donc jugé à l'aune d'un modèle qui est hors de portée, mais dont l'existence en droit est affirmée: il a existé, et il doit rester quelque part des gens qui le parlent. Ce qui est sans doute vrai dans une certaine mesure, si l'on s'entend sur les mots. Mais le plus révélateur est qu'on le dise ainsi. En somme, parler sanaani, c'est employer des mots qui ne s'emploient plus: dihlìz pour un couloir, țārūd pour une galerie.

Ces exemples, parmi les plus fréquemment cités, sont révélateurs d'un fait ; ce parler sanaani idéal est indissolublement lié à un lieu: la vieille ville de Sanaa, avec son architecture raffinée et le mode de vie qui l'accompagne ou l'accompagnait. Cette affirmation revient plusieurs fois au cours des entretiens: le vrai sanaani n'est parlé que par les gens originaires de la vieille ville, șan'ā l-gadìmih, et qui y ont vécu. Et encore, certains quartiers seulement. Une informatrice soutient même que l'eau que l'on boit influe sur le langage, et que celle de la vieille ville est particulière à cet égard.

À quoi reconnaît-on ce vrai parler sanaani? Là aussi, une même réponse revient chez la plupart des informateurs: al-mīzih. Ce terme désigne, dans un sens restreint, le fait de vouvoyer, quand on lui parle, une personne à qui l'on veut marquer du respect, ou de la désigner, quand on parle d'elle, à la troisième personne du pluriel; dans un sens plus large, mizih désigne tout l'appareil de la politesse sanaanie traditionnelle: șāhib șan'ā șāhib mìzih, yidrī kayf yimayyiz an-nās wa-yihayyin, « le Sanaani est homme de politesse, il sait employer la bonne formule au bon moment " (Maloom et al. 2009); ainsi, si l'on demande à quelqu'un de rendre un service considéré comme déplaisant (faire passer une paire de chaussures, par exemple), on doit lui dire hāō̌̌kum wa- ${ }^{\top} a z z$ gadrukum "sauf votre respect, que Dieu élève votre valeur ", à quoi il répond gadrukum 'ālē « la vôtre est élevée »; une personne qui peut difficilement se lever pour une raison ou une autre salue un arrivant en disant al-gāyim 'azīz " celui qui est debout est cher ", à quoi l'autre répond al-jālis afdal « et celui qui est assis est encore meilleur ». Pour ce qui est du vouvoiement, on peut douter qu'il soit en voie d'extinction à Sanaa, et on l'entend fréquemment employer pour s'adresser aux aînés; 
il y a donc une apparente contradiction avec l'idée que le vrai sanaani ne se parle plus. Mais les entretiens révèlent, dans le détail, des situations très variées où les règles traditionnelles (vouvoiement du mari par la femme ou des parents par les enfants) sont souvent remises en cause par des situations sociales nouvelles, en particulier l'accès des deux sexes à l'éducation secondaire et universitaire, parfois aussi l'émigration ou le mariage avec des familles originaires d'autres régions du Yémen.

Importance, donc, d'un savoir-vivre transparaissant dans la langue. Mais ce savoir-vivre est réputé inégalement réparti suivant les classes sociales traditionnelles. Les descendants du Prophète (sādih, hāšimiyyīn) et les cadis (gudāhh) ${ }^{2}$ tiennent sous ce rapport le haut du pavé, les commerçants (tujjār) leur étant inférieurs, et c'est donc chez les hāšimiyyīn qu'on nous conseille de chercher pour trouver le vrai sanaani. Un indice discriminant, selon la moitié environ des informateurs: l'intonation. Car le Sanaani, nous dit-on, allonge les mots en parlant (biyimutț hikāyatih hìn yithākā), il dit keeeyf antoooow? "comment allez-vous?" (en vouvoyant, bien sûr), et il a un ton chantant, traînant (yilhin). Selon un témoignage, les hāšimiyyinn chantent plus que les gudāh, qui chantent plus que les tujjār. Plus on est de haute condition, plus on aurait donc le chantonnement sanaani, ce ton calme, posé, et cette douceur.

Or aux yeux de ceux de nos informateurs masculins qui ont des origines dans les régions tribales autour de Sanaa, cette intonation trâ̂nante est très fortement connotée: souplesse, mollesse, efféminement. Cela sied mal à un jeune homme qui veut être un homme. L'un d'eux refuse explicitement d'être considéré comme un Sanaani, bien qu'il soit né et ait grandi en ville, pour cette raison précise; il rejette cette lahjih gamiših, ce parler suave et affecté, qu'il assigne à certains quartiers de la vieille ville ou à certaines familles - qui existent donc bel et bien à ses yeux.

Tous les témoignages ne sont pas aussi tranchés, et les contraires peuvent coexister: le parler sanaani ne fait peut-être pas très masculin, mais au moins c'est une marque de savoir-vivre. Il reste que l'opposition qu'on rencontre là a des racines anciennes: Sanaa est une hijrih, un lieu de trêve, qui jouit d'un statut d'extraterritorialité par rapport aux tribus et où la violence armée à la base de l'équilibre du droit tribal est suspendue. Elle est le siège d'un État, imamite jusqu'en 1962, républicain depuis, possédant le monopole de la violence. Le Sanaani n’a pas le

2. Il s’agit là de la catégorie sociale des cadis, et non des juges à proprement parler, bien que les cadis aient de fait fourni la majeure partie des hauts fonctionnaires du régime imamite. 
devoir qu'a le gabìli, l'homme de tribu, d'assurer lui-même par les armes l'intégrité de sa personne, de ses biens et de sa parole. D'un point de vue tribal, il souffre d'un manque de virilité. Il est protégé, comme les juifs, les barbiers et d'autres catégories sociales dominées. Comme, également, les femmes. D'un point de vue citadin, le gabili est un barbare hirsute, grossier et dangereux: gaššām șan'ā wa-lā šayh al-bilād, " mieux vaut un maraîcher de Sanaa qu'un cheikh de tribu ». Les changements économiques et sociaux ont bien sûr bouleversé ces conceptions, qui sont en pleine recomposition. Ce n'est pas pour autant qu'elles disparaissent, et le jeune homme interrogé, s'il ne prendra sans doute jamais les armes dans une guerre tribale, se réclame tout de même de ce système de valeur, au moins d'un point de vue linguistique. Quant aux Sanaanis de souche ou réputés tels, on pourrait dire que plus ils ont à gagner à se prévaloir d'une identité citadine, plus ils traînent en parlant. Plus la hiérarchie sociale traditionnelle leur est défavorable, plus il leur est profitable d'aller chercher du crédit dans une virilité de type tribal ${ }^{3}$.

\section{L'alternative? Le parler des hommes de tribus de Sanaa}

C'est peut-être à la lumière de ces considérations qu'il faut comprendre une expression employée par beaucoup de nos informateurs lahjat gabāyil $\operatorname{șan}^{\Upsilon} \bar{a}$, « le parler des hommes de tribus de Sanaa ", qui n’est pas, à leurs yeux, du vrai sanaani. Plus on descendrait dans l'échelle sociale, plus on rencontrerait cette façon de parler, plus rude, qu'ils associent souvent à la fois à une immigration rurale et à un milieu populaire, bien que les deux choses n'aient aucune raison a priori de coïncider. La différence serait marquée, entre autres, par l'emploi de certaines expressions, comme 'ayyin, " regarde ", au lieu de ibsirū, bi-rā'yikum yā rijāl pour prendre congé au lieu de nista'dinkum. Il convient d'être prudent sur l'extension réelle des usages linguistiques en question, mais ils nous ont été signalés à plusieurs reprises. Il serait intéressant d'explorer davantage l'histoire de l'immigration pré-révolutionnaire à Sanaa. Une étape importante a dû être, à partir de 1949, le départ des juifs du quartier d'al-Gā̄, où s'installèrent des familles musulmanes d'origines diverses et qui est cité aujourd'hui comme un haut lieu de la lahjat gabāyil șan'ā.

Le jeune homme dont on parlait plus haut ajoute un commentaire intéressant: d'après lui, certains jeunes gens d'extraction hāšimī modifieraient leur façon de parler, imitant les gabāyil șan'ā pour éviter

3. Les tujjār sont, à cet égard, inférieurs aux hāšimiyyīn et aux quḍāh, mais supérieurs aux artisans et surtout à ceux qui exercent ou sont censés exercer un métier considéré comme vil: coiffeurs, bouchers, maraîchers... 
les moqueries de leurs camarades. On nous a cité, à l'inverse, deux exemples de femmes originaires d'autres régions qui auraient adopté une manière de parler "sanaanie " après leur mariage, la douceur et le calme citadin convenant visiblement mieux à une femme qu' un parler rural.

Certains archaïsmes sanaanis sont aujourd'hui connotés comme féminins. Des salutations comme șbahtū, "bonjour ", et ka-msaytū, "bonjour/bonsoir ", naguère employées par tous et encore présentées comme standard par Watson (1996) nous ont été explicitement décrites par de jeunes locuteurs de famille sanaanie comme typiquement féminines. De fait, sauf parfois dans la bouche de personnes âgées, nous ne les avons que très rarement entendues en milieu masculin; on fait rire si l'on s'avise d'y avoir recours. Il est probable que leur emploi recule même en milieu féminin. Watson (2004 : 565) remarque que ces expressions sont de celles qui, chez les Sanaanis d'origine, disparaissent quand ils adoptent un outsider register en s'adressant à des interlocuteurs originaires d'ailleurs, et qu'elles sont remplacées par șabāh al-hayr et masā al-hayr.

Un parler identifiable comme originaire d'Aden a des connotations particulières. Il semble qu'il soit signe de culture et de modernité internationale, mais tout en manquant de virilité. Ainsi une informatrice originaire d'Aden et dont les deux parents sont adénis dit continuer à parler adéni en toutes circonstances, tandis que son frère est gêné de le faire en présence de ses amis sanaanis et adopte un parler de type gabāyil șan'ā. Elle dit également avoir du mal à comprendre les femmes de Sanaa quand elles parlent entre elles. Le caractère peu ocuménique du sanaani est relevé par une autre informatrice, elle-même issue d'une famille de guḍāh de Sanaa et âgée de 36 ans, qui dit que, pour se faire comprendre, elle est généralement obligée de s'écarter du sanaani:

Pour que les autres nous comprennent, nous employons beaucoup de mots égyptiens et adénis. Mais quand il s'agit de plaisanter avec des Sanaanies, alors nous parlons sanaani. (...). Le dialecte sanaani a changé, les mots, l'intonation, et ce n'est plus comme avant, sauf chez quelques personnes qui n'ont pas quitté la vieille ville et qui continuent à dire: unguși pour uskubī ["verse!»], gāmiz pour nadīif [" propre»], hālibb pour ḥâdur [" oui? ", en réponse à une interpellation].

Une autre informatrice, du même âge, issue d'une famille de guḍāh de Sanaa, est mariée à un homme d'une famille de gudāh de la région de Ibb. Sa mère est sanaanie, mais son père est en fait originaire de Tulā et a des origines tribales. Elle-même a vécu plusieurs années en Égypte avec son mari. "Je mélange ", dit-elle, «beaucoup de mots égyptiens quand 
je parle avec les autres qui ne sont pas sanaanies. (...) Avec les Sanaanies, je parle sanaani mais en mélangeant avec la lahjat gabāyil șan'ā pour ce qui est de la rudesse de l'intonation [aș-șawt al-gawī] »

Ces derniers exemples révèlent un phénomène important, que les circonstances des entretiens n'ont pas fait apparaître, sans doute, dans toute sa mesure: le développement, chez un même locuteur, de deux registres, dont l'un est destiné à l'extérieur, c'est-à-dire à la communication interdialectale. Watson (2004) note ce phénomène aussi bien pour les Sanaanis de souche que pour les nouveaux arrivants.

C'est un fait, au demeurant, que le parler d'Aden coïncide avec l'égyptien sur un certain nombre de points, au terme d'une histoire sans doute fort ancienne. Du coup, il est aujourd'hui souvent plus proche que le sanaani de ce qui est le plus standard à l'échelle du Moyen-Orient arabophone (comme yitkallam «il parle " contre yitḥākā à Sanaa, yirūh « il va » contre yisīr). Aden est la porte d'entrée de mots internationaux, comme kuwayyis "bien ", bardo " aussi " (le premier est récent mais bien implanté aujourd'hui à Sanaa, le second semble ne pas avoir dépassé Taez). En outre, durant toute la période socialiste, la capitale du Sud a été le vivier d'une classe intellectuelle tournée vers Le Caire, Moscou, Londres et très influencée dans ses pratiques langagières par l'arabe standard moderne.

\section{En guise de conclusion}

Pour conclure, constatons que le mot «șan'āni » ne sonne pas, en fait, comme une appellation géographique. Et dans le domaine de la langue, il ne désigne pas le parler d'un lieu. Il désigne avant tout un ordre social citadin, soutenu symboliquement par la figure d'un passé illustre et raffiné, et s'opposant essentiellement à un ordre tribal promouvant une figure de la virilité. Ni l'un ni l'autre de ces deux termes n'est à prendre au pied de la lettre. Certes, les nostalgiques du temps de l'Imam existent, et le vouvoiement a pu, à une certaine époque, être perçu comme anti-révolutionnaire; mais on peut fort bien - et c'est le cas général - se réclamer de l'antique urbanité sanaanie sans être pour autant partisan d'un retour à un ordre ancien. Du haut d'un toit de la vieille ville, un regard à la ville nouvelle suffit pour comprendre que le passé ne reviendra plus; mais ce n'est pas grave, car si les familles les plus proches de l'Imam ont été quelque peu écartées des bienfaits du pouvoir, d'autres grands noms de Sanaa n'ont pas eu à se plaindre du régime qui vacille ces jours-ci, et sont souvent luxueusement installés hors les murs de l'ancienne cité. Quant à ceux qui revendiquent une origine tribale en 
n'allongeant pas les syllabes, ils sont nombreux, en fait, à n'avoir jamais connu que la ville et à n'espérer rien d'autre que d'y vivre mieux. Pour combien d'entre eux la solidarité tribale lignagère est-elle réellement déterminante, plus que leur condition urbaine? C'est pour eux qu'on a créé le curieux attelage gabāyil șan'ā, qui aurait sans doute laissé bien perplexe un locuteur des années 1950. Et la façon de parler qu’on leur reconnaît est un moyen de préserver une identité gabilì sans pour autant parler comme leurs pères, en se reconnaissant implicitement comme citadins à défaut d'être « sanaanis ».

S’il s'avérait que le terme lahjat gabāyil șan`ā recouvrait une réalité linguistique concrète, alors ce serait là sans doute ce qui ressemble le plus à une koinè sanaanie, pour les hommes du moins. En effet, cette façon de parler attire à la fois des enfants de migrants issus d'un milieu linguistiquement distinct et des fils de familles sanaanies. Intuitivement, nous serions tentés de dire que la lahjat gabāyil șan'ā existe, c'est-à-dire qu'il s'agit d'un usage relativement cohérent, et nous voyons bien, en gros, ce que veulent dire nos informateurs quand ils emploient ce terme ${ }^{4}$. Mais il faudrait définir précisément en quoi elle consiste. Pour les femmes, cette expression n'est pas employée. Mais on a vu que certaines adoptaient un parler qualifié de sanaani. Que faut-il entendre par-là? Ce parler est-il très différent de la lahjat gabāyil șan'ā si l'on fait abstraction de l'intonation, virile ou féminine, qui est discriminante aux yeux de la plupart des informateurs? La réponse doit attendres.

Redisons, pour finir, que l'origine septentrionale des informateurs a pu jouer dans les résultats obtenus. Ainsi, il est frappant de remarquer que les caractéristiques du sanaani qu'on nous a fournies sont presque toujours les mêmes: quelques lexèmes désignant des réalités obsolètes, le vouvoiement, l'intonation traînante. C'est à peu près tout. Rien qui concerne l'articulation d'un phonème donné, ou une particularité morphologique. Il en aurait peut-être été autrement si l'on avait interrogé davantage de gens originaires de la Hujariyyah, province qui se retrouve une fois de plus sous-étudiée en dépit de son rôle capital.

Le tableau est donc loin d'être achevé.

4. Nous ne pouvons pas suivre Freeman (2006) dans ses conclusions, $c f$. Dufour 2008.

5. Cf. cependant l'étude de Watson (2007) sur les différences de glottalisation pausale entre Sanaanis d'origine et d'adoption. Ce phénomène ne relève pas à proprement parler de l'intonation, mais il lui est intimement lié. 


\section{Références bibliographiques}

Behnstedt P. (1985), Die nordjemenitischen Dialekte. Teil 1: Atlas, Wiesbaden, Dr. Ludwig Reichert Verlag.

Dufour J. (2008), Attitudes linguistiques et identités locales à Șan`â?, Revue des mondes musulmans et de la Méditerranée, 121-122: YémenTerritoires et identités, p. 133-144, disponible sur remmm.revues.org/ index4893.html?file $=1$.

Freeman A. (2006), Why there is no koiné in San'aa?, Yemen, in S. Boudelaa (ed.), Perspectives on Arabic Linguistics, vol. xvi: Papers from the sixteenth annual symposium on Arabic linguistics, Cambridge, March 2002, Amsterdam/Philadelphie, John Benjamins Publishing Company (p. 63-82).

Maloom H., Dufour J. \& Dumas F. (2009), Expression de la politesse et de la civilité dans la société sanaanie, Estudios de dialectología norteafricana y andalusí, 13, p. 121-152.

Rossi E. (1938), Appunti di dialettologia del Yemen, Rivista degli studi orientali, XVII, p. 230-314.

- (1939), L'arabo parlato a Șan' $\bar{a}^{2}$, Rome, Istituto per l'Oriente.

Stadnicki R. (2009), Nouvelles centralités et recompositions socio-spatiales dans le grand Sanaa (Yémen), thèse de doctorat, Tours, Université François Rabelais, disponible sur: http://tel.archives-ouvertes.fr/docs/00/44/94/ 84/PDF/R._Stadnicki_2009_Nouvelles_centralites_et_recompositions_socio-spatiales_dans_le_Grand_Sanaa_Yemen_.pdf

Watson J.C.E. (1993), A Syntax of Șañānī Arabic, Wiesbaden, Harrassowitz.

- (1994), On the definition of dialect with reference to Yemeni dialects of Arabic, in Y. Suleiman (ed.), Arabic Sociolinguistics. Issues \& Perspectives, Richmond (Angleterre), Curzon Press (p. 237-250).

- (1996), Șbaḥtū! A Course in Șan'ānī Arabic, Wiesbaden, Harrassowitz.

- (2004), Insiders, outsiders, and language development in San'a, in I. Ferrando \& J. J. Sanchez Sandoval (eds), AIDA $5^{\text {th }}$ Conference Proceedings, Cadiz, septembre 2002, Cadix, Servicio de Publicaciones Universidad de Cadiz (p. 561-70).

- (2007), Linguistic leveling in San'ani Arabic as reflected in a popular radio serial, in C. Miller, E. Al-Wer, D. Caubet \& J.C.E. Watson (eds), Arabic in the City, Issues in dialect contact and language variation, Londres/New York, Routledge (p. 166-187). 